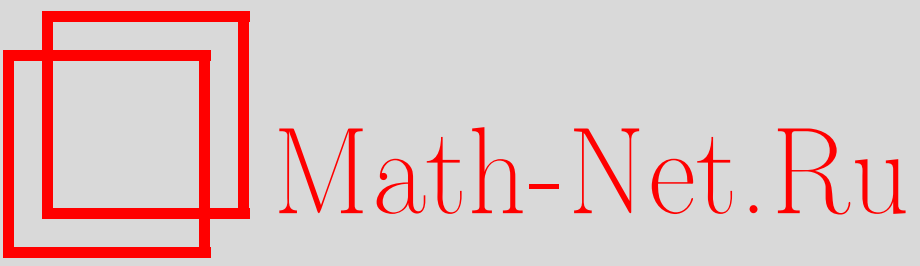

Н. Г. Кружилин, Голоморфные автоморфизмы двумерных гиперболических трубчатых областей, УМH, 2004, том 59, выпуск 5, 155-156

DOI: https://doi.org/10.4213/rm781

Использование Общероссийского математического портала Math-Net.Ru подразумевает, что вы прочитали и согласны с пользовательским соглашением

http://www.mathnet.ru/rus/agreement

Параметры загрузки:

IP: 54.197 .130 .99

26 апреля 2023 г., 13:38:58 


\title{
ГОЛОМОРФНЫЕ АВТОМОРФИЗМЫ ДВУМЕРНЫХ ГИПЕРБОЛИЧЕСКИХ ТРУБЧАТЫХ ОБЛАСТЕЙ
}

\author{
Н. Г. Кружилин
}

Пусть $\Omega$ - область в $\mathbb{R}^{2}$. Трубчатой областью с базой $\Omega$ называется область $D(\Omega)$ в $\mathbb{C}^{2}$ следующего вида: $D(\Omega)=\left\{z=(x+i y) \in \mathbb{C}^{2}: y \in \Omega\right\}$. Всякая трубчатая область переводится в себя сдвигами $z \mapsto z+v, v \in \mathbb{R}^{2}$. Кроме того, если существуют вещественные афффинные отображения, отображающие базу в себя, то они естественно продолжаются до афффинных голоморфных автоморфизмов самой области. При этом, если область не является прямым произведением комплексного линейного пространства и трубчатой области меньшей размерности, то других аффинных автоморфизмов вида у нее нет. Простейшие примеры трубчатых областей с неаффинными голоморфными автоморфизмами - это верхняя полуплоскость $\mathbb{C}_{+}$и горизонтальная полоса в $\mathbb{C}$ (голоморфно эквивалентные друг другу).

Среди трубчатых областей с ограниченной базой неаффинные автоморфизмы имеются только у областей, имеющих вид прямого произведения области меньшей размерности на некоторую степень (скажем, $k$ ) полосы (см. [1]). Соответственно, группы их автоморфизмов $\operatorname{Aut}(D)$ представимы в виде прямого произведения группы аффинных автоморфизмов первого сомножителя и группы голоморфных автоморфизмов второго (изоморфной $\left.\mathrm{Aut}\left(\mathbb{C}_{+}\right)^{k} \times S_{k}\right)$.

В двумерном случае из работы [2] можно извлечь также описание голоморфных автоморфизмов трубчатых областей над выпуклой базой, не содержащей полных прямых.

Мы описьваем голоморфные автоморфизмы более широкого класса гиперболических (по Кобаяси) двумерных трубчатых областей. Это описание выражается следующим результатом.

Теорема. Пусть $D$ - гиперболическая трубчатая область в $\mathbb{C}^{2}$ с основанием $\Omega$. Тогда либо все голоморфные автоморфизмы $D$ аффинные, либо $D$ аффинно әквивалентна трубчатой области с базой, имеющей один из перечисленных ниже видов:

(1) $\quad\left\{y_{2}>y_{1}^{2}\right\}$;

(2) $\quad\left\{y_{1}>0, y_{2}>0\right\}$

(3) $\quad\left\{0<y_{1}<\pi, 0<y_{2}<\infty\right\}$;

(4) $\quad\left\{0<y_{1}<\pi, 0<y_{2}<\pi\right\}$;

(5) (a) $\left\{y_{2}>-\ln \sin \left(y_{1}\right), 0<y_{1}<\pi\right\}$;

(b) $\left\{y_{2}<-\ln \sin \left(y_{1}\right), 0<y_{1}<\pi\right\}$;

(c) $\left\{-\ln \sin \left(y_{1}\right)<y_{2}<-\ln \sin \left(y_{1}\right)+s, 0<y_{1}<\pi\right\} ; s>0$;

(6) (a) $\left\{y_{2}>e^{y_{1}}\right\}$;

(b) $\left\{0<y_{2}<e^{y_{1}}\right\}$;

(c) $\left\{e^{y_{1}}<y_{2}<h e^{y_{1}}\right\} ; h>1$.

Все указанные области, за исключением первой, были включены в [3] в списки гипреболических трубчатых областей, являющихся голоморфно, но не аффинно эквивалентньми.

Отображение $z_{1}^{*}=z_{1}, z_{2}^{*}=z_{2}+\frac{i}{2} z_{1}^{2}$ переводит область (1) в стандартную неограниченную реализацию единичного шара $\left\{y_{2}>\left|z_{1}\right|^{2}\right\}$, так что группа голоморфиных автоморфизмов этой области 8-мерна, а формулы для ее автоморфизмов могут быть получены соответствующим преобразованием общеизвестных формул для дробно-линейных автоморфизмов шара.

Области (2), (3) и (4) очевидно биголоморфно эквивалентны. Каждая из компонент произвольного голоморфного автоморфизма области (2) - это дробно-линейное отображение, зависящее от одного из переменных, так что $\operatorname{Aut}(D)=\operatorname{Aut}\left(\mathbb{C}_{+}\right)^{2} \times \mathbb{Z}_{2}$ имеет размерность 6.

Как показано в [2], компонента единицы группы автоморфизмов области (6)(а) образована отображениями вида $z_{2} \mapsto \frac{a z_{2}+b}{c z_{2}+d}\left(\left(\begin{array}{ll}a & b \\ c & d\end{array}\right) \in \mathrm{SL}(2, \mathbb{R})\right), z_{1} \mapsto z_{1}-2 i \log \left(c z_{2}+d\right)+t(t \in \mathbb{R})$, так что она 4 -мерна и изоморфна $\operatorname{Aut}\left(\mathbb{C}_{+}\right) \times \mathbb{R}$, где второй сомножитель соответствует сдвигам

Работа выполнена при поддержке РФФИ (грант № 02-01-01291) и программы поддержки ведущих научных школ РФ (грантНШ-2040.2003.1). 
по $x_{1}$. Всякое отображение $\phi \in \operatorname{Aut}(D)$ индуцирует сопряжением некоторьй групповой автоморфизм компоненты единицы, причем подправив его внутренними автоморфизмами компоненты единицы, т.е. не меняя компоненты связности $\operatorname{Aut}(D)$, содержащей $\phi$, мы можем считать, что подгруппа сдвигов по $x$-направлениям переходит в себя. Но в этом случае $\phi$-афффинное отображение, а легко видеть, что все аффинные автоморфизмы области (6)(а) описьваются приведенными выше формулами и потому лежат в компоненте единицы полной группы. Следовательно, $\operatorname{Aut}(D)$ для области (6)(a) в действительности связна и все ее элементы описаны выше.

Несложно показать, что области $(6)(\mathrm{b})$ и $(6)(\mathrm{c})$ имеют в точности те же голоморфные автоморфизмы. Вдобавок, соответствующие области типов (5) и (6) голоморфно эквивалентны посредством отображения $z_{1}^{*}=i z_{1}-z_{2}, z_{2}^{*}=\exp z_{1}$ (см. [2]). Таким образом, их группы голоморфных автоморфизмов изоморфны, а сами автоморфизмы областей типа (5) также допускают явное описание, хотя и несколшко более громоздкое.

Переходя к доказательству теоремы, напомним, что в рассматриваемом случае группа голоморфных автоморфизмов $\operatorname{Aut}(D)$ является группой Ли, двумерная группа сдвигов в чисто вещественных направлениях является ее максимальной связной коммутативной подгруппой, и всякий автоморфизм $D$, сохраняющий действие этой подгруппы, - аффиннньй. Таким образом, при $\operatorname{dim} \operatorname{Aut}(D)=2$ доказьвать нечего. Как отмечено в [3], трубчатые области с $\operatorname{dim} \operatorname{Aut}(D) \geqslant 5$ найдены в [2]: это области типов $(2),(3)$ и (4) в теореме. Области с $\operatorname{dim} \operatorname{Aut}(D)=3$ или 4 также уже рассматривалимь в [3]. Однако в первом случае там формально не конца исследована ситуация, когда трехмерная группа $\operatorname{Aut}(D)$ имеет двумерньй коммутант, хотя и отмечено что в этом случае группа сдвигов во вполне вещественных направлениях - единственная связная двумерная подгруппа Aut $(D)$. На самом деле тогда всякий голоморфньй автоморфизм сохраняет действие этой подгруппы и $\operatorname{Aut}(D)$ состоит из афффинных отображений.

В размерности 4 не до конца исследован в [3] (гипотетический) случай группы $\operatorname{Aut}(D)$, алгебра Ли $\mathfrak{g}$ которой (состоящая из голоморфных векторных полей в $\Omega$ ) имеет образующие $L_{1}, L_{2}$, $a_{1}, a_{2}$, связанные соотношениями $\left(L_{1}, L_{2}\right)=0,\left(L_{1}, a_{1}\right)=a_{1},\left(L_{1}, a_{2}\right)=a_{2},\left(L_{2}, a_{1}\right)=a_{2}$, $\left(L_{2}, a_{2}\right)=-a_{1},\left(a_{1}, a_{2}\right)=0$, где $L_{j}$ векторные поля с $(1,0)$-компонентами вида $\partial / \partial z_{j}$.

Решая в этом случае соответствующие простые системы ОДУ, мы видим, что векторные поля $a_{1}$ и $a_{2}$ продолжаются на все комплексное пространство. В силу их голоморфиности $a_{1}$ и $a_{2}$ либо всюду комплексно линейно зависимы, либо линейно независимы вне аналитического подмножества. Первьй вариант невозможен, потому что иначе мы получили бы в $D$ голоморфиные кривые с двумерной абелевой группой автоморфизмов и потому негиперболические.

Поскольку граница трубчатой области $D$ не может быть аналитическим множеством, на ней есть двумерная вполне вещественная орбита $O$ алгебры $\left(a_{1}, a_{2}\right)$. Граница $D$ не может быть и полномерной, поэтому существует линейная комбинация $L$ векторов $L_{1}$ и $L_{2}$, лежащая в касательном пространстве к $O$ в некоторой точке орбиты, а значит, орбита этой точки относительно 3 -мерной алгебры $\left(a_{1}, a_{2}, L\right)$ совпадает с $O$. Ее можно накрыть стандартной плоскостью так, чтобы $a_{1}$ и $a_{2}$ соответствовали стандартные координатные векторные поля на плоскости. Тогда $L$ будет соответствовать афффинное векторное поле на этой плоскости, линейная часть которого имеет ненулевые собственные значения, так что поле $L$ с необходимостью обращается в нуль на $O$, что противоречит его определению.

Таким образом, в случае гиперболической трубчатой области группа $\operatorname{Aut}(D)$ не может иметь указанную выше структуру и доказательство теоремы завершено.

\section{СПИСОК ЛИТЕРАТУРЫ}

[1] S. Shimizu // Math. Ann. 1999. V. 315. P. 295-320. [2] S. Shimizu // Amer. J. Math. 2000. V. 122. Р. 1289-1308. [3] Н. Г. Кружилин, П. А. Солдаткин // Матем. заметки. 2004. T. 75. № 5. C. 187-222. 